\title{
Diagnostic procedure in bleeding of obscure origin from the alimentary canal
}

\author{
FRANZ BÁRÁNY AND LARS H:SON NILSSON \\ From the Department of Gastroenterology, St Erik's Hospital, Stockholm, Sweden
}

SUMMARY The diagnostic methods used in cases of melaena where the history and clinical picture provide no evidence of the site of bleeding are surveyed. In the light of our own experience with a series of 52 cases the most suitable examination procedure is discussed in detail.

Endoscopy and barium radiological examinations of the gastrointestinal tract have long been relied upon to locate the site of gastrointestinal bleeding, but in many instances in large series these routine methods have been of no assistance (Rives and Emmett, 1954; Birke and Engstedt, 1956; Jones, Read, and Stubbe, 1959). Over the years a number of special techniques intended to increase the chances of finding the source of bleeding have been described.

\section{Radiological Techniques}

The small intestine 'enema' is a radiographical technique which allows a more reliable consecutive examination of the various sections of the small intestine than the conventional passage of contrast medium. First described by Abrams and Jarvis (1912), this method gained fairly wide use in the 1920s (eg Pesquera, 1929) but then fell more or less into oblivion until it quite recently experienced a renaissance (Harden, 1960; Pygott, Street, Shellshear, and Rhodes, 1960).

The barium-air, double-contrast method for the visualization of polyps and similar changes in the colon wall (Welin, 1958) has proved to be of considerable value, and, at least in Sweden, has been widely applied in cases of haemorrhage.

In the last three years selective angiography of the arteries of the stomach and bowel has been used successfully for the location of bleeding (Baum, Nusbaum, Blakemore, and Finkelstein, 1965; Boijsen and Reuter, 1967; Reuter and Book- stein, 1968; Halpern, Turner, and Citron, 1968) but the amount of published material is still too small to provide a reliable basis for an evaluation of the definitive value of this use of the method.

A special type of small intestine enema is hypotonic duodenography using the method of Liotta (1955). Here a considerable inhibition of the duodenal peristalsis is produced by an intravenous injection of a fairly large dose of an anticholinergic agent. During the subsequent paresis the duodenum is filled with contrast medium through a tube, though the doublecontrast technique may also be applied. The method is now used to some extent in Scandinavia (Stage and Banke, 1968), and it might be of value in the case of suspected haemorrhage in the duodenum, caused, for instance, by a tumour of the pancreas or duodenal papilla.

\section{Intubation Technique}

The intubation technique has been used to locate the site of haemorrhage. Einhorn's string test (Einhorn, 1909) has the advantage that it permits of the detection and location of short, intermittent bleeding that may occur while the string is in place. Too often, unfortunately, this test gives false positive results because the string itself damages the mucosa (Smith, 1961); in our experience such lesions of the cardia are the rule rather than the exception. As this is not the case when a plastic tube is used, the injury to the cardia must be ascribed to the string. By enclosing the string in a plastic tube (Valtonen, 1967) 
having a large number of holes along its length it should be possible to eliminate this serious disadvantage of the string test.

Intravenous injection of fluorescein in combination with the string test has been recommended as a more reliable method (Traphagen and Karlan, 1958; Haynes, Pittman, and Christakis, 1960). Fluorescein can be demonstrated in the bile only seven to eight minutes after its injection (Pittman, 1964); if any fluorescein and blood on the string distally to the pylorus are to be taken as a reliable indication of current bleeding the string must be drawn up within a few minutes of the injection. It is difficult to see any essential advantage in using fluorescein; it is true that in the occasional case a positive reaction may directly disclose bleeding as still going on. On the other hand, the fluorescein test will give a high proportion of false negative results, since in most cases intestinal bleeding is intermittent.

Intubation with a tube through which a specimen of the intestinal contents can be aspirated is a method which, since it was first described by Miller and Abbott in 1934, might well have come to be adopted as a routine method, but would still seem to be used only in a fraction of all cases of melaena presenting difficulty in diagnosis. It is often an extremely time-consuming examination. A small balloon, or the like, at the tip of the tube would allow the intestine to take a hold. Even then it usually takes two to three days before the tube has been carried down to the distal part of the ileum. To facilitate normal eating in the meantime and to avoid the risk of damage to the tube through chewing, it is preferable to introduce it through a nostril rather than through the mouth.

During intubation fluoroscopic observation is kept to see that tube is not curling up in the stomach. To diminish the risk of this happening the patient should be instructed to fix the tube at meals to avoid it being fed down through the oesophagus during swallowing. Just after meals, on the other hand, the tube should be released, so that any propulsive motor action in the stomach or bowel will carry the balloon attached to the tip of the tube downwards.

The tube should be made of a plastic material that softens slightly at body temperaturepolyvinyl, for instance; a suitable outer diameter is $2.5 \mathrm{~mm}$ to $3 \mathrm{~mm}$, with a wall thickness of about 0.4 millimetres. A tube of this type causes less discomfort during prolonged intubation than does a rubber tube. It should be marked to show the distance to the tip, preferably in such a way as to be visualized during radiological control, or the tube may, of course, be filled with a contrast medium. The balloon at the tip should contain an opacifying medium.

Green (1964) writes: 'The disadvantage of these tubes is that they cannot deliver small amounts of fluid from all levels of the bowel. Unless the amount of fluid exceeds the capacity of the tube it must traverse, intestinal muscosa blocks the lumen, and the fluid stays in the tube until another pool of intestinal fluid allows the mucosa to drift away from the tube's orifice. This dead space in the tube can present severe $\vec{\Rightarrow}$ difficulties in a test such as this since . . . fluid $\stackrel{5}{\stackrel{9}{9}}$ entering the end of the tube at a given level in the gut may not be delivered from the other end until the tube has moved much farther $\frac{\vec{\sigma}}{\vec{D}}$ down.' Crosby, Crosby, and Kugler (1960) $\stackrel{ه}{\circ}$ have described a capsule that, inserted in the is tip of a double-lumen catheter, can serve as a $\overrightarrow{0}$ bubble trap, thereby eliminating the problems produced by a too large dead space. Green $\vec{\omega}$ (1964) has designed a simplified modification of this capsule.

The aspirate is analysed for blood pigment. Radioactive labelling of blood corpuscles in connexion with the intubation technique has $\mathcal{D}_{-}$ been recommended for determining the site 0 of gastrointestinal bleeding (Ebaugh, Clemens, Rodnan, and Petersen, 1958; Pillow, Hill, $\vec{\longrightarrow}$ Ragen, Siemsen, and Walker, 1962), but it is difficult to see its advantage for this purpose, though it can certainly help to ascertain the $\vec{\theta}$ magnitude of the haemorrhage (Gerritsen, Heinz, and Stafford, 1954; Bannerman, 1957; Roche, Perez-Giminez, Layrisse, and Di Prisco, 1957).

With the plastic tube having a number of lateral holes near the tip and a terminal balloon $\stackrel{\mathbb{D}}{\circ}$ as already described, false positive results are $\overrightarrow{\vec{B}}$ rare. A recommended procedure for obtaining the most accurate location of the site of bleeding, when blood is found in the aspirate, will be described below. A negative result of intubation can also provide useful information, namely, that the source of bleeding probably lies distally to the tube; in this instance it is most important to repeat the aspiration procedure often and over a long enough period to ensure that the bleeding had not ceased when the tip of the tube had reached its most caudal position.

\section{Diagnostic Laparotomy}

An operation will be necessary sooner or later in most cases of intestinal bleeding, except in cases where the history or blood examinations $\stackrel{\oplus}{\rightarrow}$ point to a haemorrhagic diathesis, or, sometimes, 0 where there are inflammatory changes or extensive haemangiomatosis. When the source of bleeding has been located either by radiology or intubation, laparotomy should as a rule be performed without delay, when the surgeon should have little difficulty in finding the alteration responsible for the haemorrhage.

There is no standard answer to the question when laparotomy shall be performed in cases $P$ in which it has not been possible to locate the bleeding. The chance of being unable to find 
the source of bleeding at the operation must be weighed against the risk of a perhaps fatal delay in instituting treatment. The former eventuality is great if the bleeding should have ceased before laparotomy is undertaken, but is much less if the operation is conducted while bleeding is still occurring (Bockus, Ferguson, Thompson, and Roth, 1953; Stahlgren and Ferguson, 1958).

Laparotomy will hardly be undertaken before a conventional radiological examination of the gastrointestinal tract. If this examination has been uninformative and the bleeding continues, immediate angiography of the intestinal tract would probably be the best move. If angiography yields a negative result, intubation will clearly be justified; but even with positive angiograms a successful intubation can be of value, for the surgeon will then have information on both the vascular and the intestinal topography of the source of bleeding.

If the bleeding has ceased during the course of the radiological examination, as commonly happens, the next step can be one of the following. Any radiological diagnostic procedure which should certainly be carried through should include a barium-air, double-contrast enema, angiography, possibly also a small intestine enema, and hypotonic duodenography. The possibility that the source of bleeding may lie in the oesophagus, stomach, or rectum must be considered again and, where possible, ruled out by endoscopy. In cases in which the site remains undetected the best course is probably to await renewed bleeding but, when this happens, to act according to a preconcerted plan. The patient should be re-admitted immediately and either laparotomy or intubation performed. We prefer to delay further the surgical intervention until, at least one well planned attempt has been made to locate the source of bleeding by intubation. There is, however, no objective proof that this approach will afford a better result than immediate laparotomy performed during bleeding on the second admission.

If, at laparotomy, the source of bleeding is not established immediately by direct inspection and palpation, an examination of the small intestine by transillumination is recommended, the loops being carefully inspected in turn by viewing against an adjustable lamp placed near the abdomen. The beam should be parallel.

\section{Diagnostic Techniques under Development}

There are descriptions of technically advanced telemetric methods for demonstrating bleeding and for locating the site of bleeding. These rely on isotope-labelled blood corpuscles, or on the generation of heat when certain chemical substances make contact with constituents of the blood (Mackay, 1968); however, so far as we have found, these have not been submitted to clinical testing.

\section{Method of Intubation for the Present Series}

The following intubation technique was used in the patients forming the series of this study. A polyvinyl tube $350 \mathrm{~cm}$ long, with outer and inner diameters of 2.54 and $1.70 \mathrm{~mm}$ respectively, is furnished at one end with lateral holes through which specimens of the bowel contents can be aspirated. To the perforated end is attached a rubber balloon containing $0 \cdot 5-1 \cdot 0 \mathrm{ml}$ of mercury or $1-2 \mathrm{ml}$ of barium sulphate suspension ${ }^{1}$. The balloon should only be partly filled so that it can be passed through a nostril. The balloon serves as a sinker for the tube and facilitates its propulsion through the gastrointestinal tract.

Specimens can often be obtained by using the siphon principle. As a rule, however, in the distal part of the ileum fairly powerful suction, for instance, by means of a tight $20 \mathrm{ml}$ injection syringe, must be applied. Two samples are taken at each level and the aspirated fluid is examined immediately for the presence of blood. If none is found the tube should be allowed to advance $20 \mathrm{~cm}$ or so, the sampling then being repeated until blood is detected. The tube must now be withdrawn until negative samples are obtained to be sure that the site of bleeding is not located any great distance orally of the site of aspiration. By obtaining a number of negative and positive samples within a distance of a few decimetres the site of bleeding can be established with a fairly high degree of accuracy. Having located the level at which the bleeding occurs, the contrast medium is first injected through the tube to identify the section of the bowel. Contrast medium is then given by mouth or by the ScottHarden technique. Allowing for regurgitation the level where blood is found in the bowel may be $20 \mathrm{~cm}$ or so above the actual site of bleeding (Ferguson, 1959).

If only negative specimens are obtained from the tube during its passage down to the ileocaecal region, although there is still blood in the faeces, it is of great value to leave the tube in place for up to a week in a position where specimens can still be obtained. Intestinal bleeding usually appears to be intermittent, so that the chance of detecting it increases with the period of intubation. To the same end, moderate heparinization may sometimes be useful. The stools should be examined daily for blood, and negative aspiration specimens but positive faecal tests may be taken as a sign that the site of bleeding is distal to the tip of the tube. As will be seen from the results, it is, however, often more difficult than

${ }^{1}$ The tube used is manufactured and marketed by A. B. Louis Gibeck, 13012 Älta, Sweden. 


\begin{tabular}{|c|c|c|c|c|c|c|}
\hline \multirow[t]{2}{*}{ Site of Bleeding } & \multirow{2}{*}{$\begin{array}{l}\text { Number of } \\
\text { Patients }\end{array}$} & \multirow[t]{2}{*}{ No Laparotomy } & \multicolumn{3}{|c|}{ Laparotomy in Relation to Intubation 1} & \multirow{2}{*}{$\begin{array}{l}\text { Cause of Bleeding } \\
\text { Found at Necropsy }\end{array}$} \\
\hline & & & Before & After & Before and After & \\
\hline Still unknown & 3 & 1 & & 1 & $1 \mathrm{M}$ & \\
\hline $\begin{array}{l}\text { Small intestine } \\
<1 \text { m distal to pylorus }\end{array}$ & 15 & & & $\begin{array}{l}3 \text { BH } 2 \text { BL } 2 \text { BA } \\
3 \text { MP } 1 \text { MS }\end{array}$ & $\begin{array}{l}2 \mathrm{IO} \\
1 \mathrm{BL} 1 \mathrm{BA}\end{array}$ & \\
\hline$>1 \mathrm{~m}$ distal to pylorus & 8 & & & 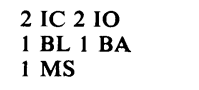 & & $1 \mathrm{IO}$ \\
\hline Caecum and ascending colon & 5 & & & $\begin{array}{ll}1 & \mathrm{IC}^{2} \\
2 & \mathrm{BH} \\
2 & \mathrm{ML}\end{array}$ & & \\
\hline Coagulation defect & 5 & 2 & 1 & 2 & & \\
\hline Total & 36 & 3 & 1 & 26 & 5 & 1 \\
\hline
\end{tabular}

Table I Positive results of intubation test (blood found in aspirate) in 36 patients

${ }^{1}$ IC stands for Crohn's disease ( 3 patients), IO for other non-tumorous disease ( 5 patients); BH, BL, and BA for benign haemangioma, leiomyoma, and adenoma (13 patients). $\mathbf{M}$ indicates malignancy proved but no primary tumour found (1 patient). MP-pancreatic carcinoma, MS - carcinoma of small intestine, and ML-carcinoma of large intestine ( 7 pat ients in all in the last three groups).

${ }^{2}$ False positive result in one patient who had Crohn's disease of the colon but blood in aspirates from lower small intest ine.

this to differentiate between proximal bleeding that has ceased, and a site of bleeding distal to the tube. Some possibilities of increasing the reliability of this technique are discussed below.

\section{Present Series}

An account is given below of 52 patients in whom an attempt was made to locate the site of bleeding by the intubation procedure already described. The series comprised all patients in whom, over the last 10 years, the usual radiological examinations and endoscopy performed before intubation yielded no information on the origin of the haemorrhage. Patients with bleeding from diverticula in the distal part of the colon, in whom intubation was also performed to rule out a more proximal location of the bleeding, have not been included in the present series, which consisted of 27 men and 25 women, ranging in age from 19 to 80 years (mean 55). As a rule several earlier unsuccessful attempts, without intubation, had been made to ascertain the site of bleeding.

Of the patients in the series, four had not been admitted previously; 10 patients had been admitted once before; 10 twice before; 16 between three and five times before; nine patients had been admitted between six and 10 times previously; and three patients had a history of more than 10 admissions. In nine patients the interval between the first occasion of diagnosed bleeding and intubation ranged from one week to six months. In six patients this interval was between seven and 12 months; in 13 between one and two years; in a further 13 between two and five years; and in 11 patients the interval was more than five years. Before intubation the patients with subsequently confirmed malignant tumours had been admitted to hospital for haemorrhage on average 3.0 times and the average length of the history was 1.75 years. For the patients with non-malignant diseases the corresponding figures were 5.7 admissions and 4.05 years' history. Nine patients had undergone unsuccessful diagnostic laparotomy before intubation.

The data and results for the 36 patients in whom blood was detected in aspirates from the intubation tube are presented in Table I. In 15 patients the bleeding was located in the upper part of the small intestine. In the subsequent laparotomy nine of these were found to have a benign and four a malignant tumour as the cause of the bleeding. In one case an area of the jejunum between 20 and $30 \mathrm{~cm}$ long contained diffuse polypous hyperplasia of the mucosa that could not be more accurately classified histologically. In one case the bleeding was due to choledocholithiasis with subacute cholecystitis. This as the source of bleeding had escaped detection at an earlier laparotomy.

In eight cases the source of bleeding was situated in the small intestine more than 1 metre distally to the pylorus. Three of these patients had neoplastic disease and one had a Meckel's diverticulum containing an ulcer. In the remaining four patients the cause of bleeding was inflammatory or vascular. In five patients blood was detected in aspirates from the ileocaecal region. Two of them had haemangiomatosis and two had carcinoma. One patient had regional enteritis located in the ascending and transverse colon; blood was found in the aspirates when the tube was located several decimeters proximal to the ileocaecal valve in spite of the fact that at laparotomy the small intestine was devoid of 


\begin{tabular}{|c|c|c|c|c|c|c|c|}
\hline \multirow[t]{3}{*}{ Site of Bleeding } & \multirow{3}{*}{$\begin{array}{l}\text { Number of } \\
\text { Patients }\end{array}$} & \multicolumn{3}{|c|}{ Result of Intubation ${ }^{1}$} & \multicolumn{3}{|c|}{ Cause of Bleeding } \\
\hline & & \multirow[t]{2}{*}{ Correct } & \multirow[t]{2}{*}{ False } & \multirow[t]{2}{*}{ Doubtful } & \multicolumn{3}{|c|}{ Laparotomy after Intubation At Necropsy } \\
\hline & & & & & Located & Not Located & \\
\hline Unknown & 6 & & & $\begin{array}{l}5 \mathrm{ND} \\
1 \mathrm{M}\end{array}$ & 1 & 3 & \\
\hline Stomach & 3 & & $\begin{array}{l}1 \mathrm{GU} \\
2 \mathrm{GM}\end{array}$ & & $\begin{array}{l}1 \\
2\end{array}$ & & \\
\hline Small intestine & 2 & 110 & $1 \mathrm{MS} \dagger$ & & $\begin{array}{l}1 \\
1\end{array}$ & & \\
\hline $\begin{array}{l}\text { Caecum and } \\
\text { ascending colon }\end{array}$ & 4 & $\begin{array}{l}1 \text { IO } \\
1 \text { BH } 1 \mathrm{BA} \\
1 \mathrm{ML}\end{array}$ & & & $\begin{array}{l}1 \\
1 \\
1\end{array}$ & & 1 \\
\hline $\begin{array}{l}\text { Colon distal to } \\
\text { hepatic flexure }\end{array}$ & 1 & 1 BA & & & & & \\
\hline Total & 16 & 6 & 4 & 6 & & & \\
\hline
\end{tabular}

Table II Negative result of intubation test in 16 patients

${ }^{1}$ ND means diagnosis not revealed (5 patients), GU-gastric ulcer (1 patient), GM-gastric carcinoma ( 2 patients). The other notations are as in Table I. There were 2 IO patients, 2 BA patients, and BH, M, MS, and ML (one patient each).

'Polyp diagnosed at second barium enema performed after intubation.

gross changes. This is the only 'false' positive result in the series.

Intestinal bleeding was the only manifestation of disease in five other patients with a coagulation defect; four of them had von Willebrand's disease and one had a rare form of haemophilia, probably with a disturbance of thromboplastin synthesis. During the long period elapsing until the diagnosis two of these five patients had undergone laparotomy.

In spite of the indication as to the site of bleeding obtained by intubation, in three cases the nature of the source was still obscure. One patient had been submitted unsuccessfully to diagnostic laparotomy on three occasions (195859) before intubation, which was performed in 1961, but it was a further two years before laparotomy was repeated. The abdomen presented extensive carcinosis, but although the primary tumour could not be found, it seems safe to ascribe the bleeding to the malignant disease. Another patient had bled repeatedly and unsuccessful diagnostic laparotomy was performed four years after intubation. In a third patient, who had not been operated on four years after intubation, bleeding had not recurred.

The data and results for the 16 patients in whom blood had not been detected in the aspirates during intubation are presented in Table II. In six patients there was bleeding distal to the lowest level of the tube. One of these patients had carcinoma of the caecum, three had benign tumours of the colon, one had a unique mucosal and vascular change in the caecum that defied classification, and one had a Meckel's diverticulum with an ulcer. In this last patient the bariumfilled bladder at the tip of the tube caught in the region of the ulcer, thereby providing unexpected diagnostic information.

In four patients the interpretation of the intubation results must be regarded as incorrect. None of the aspirates contained blood, but the guaiac test on faeces specimens was still positive several days after the tip of the tube had reached the distal part of the ileum, and it was concluded that the site of bleeding was situated distally to the ileocaecal valve. Two of the patients, however, had gastric carcinoma; one had a large chronic peptic ulcer high up on the dorsal wall of the stomach near the lesser curvature; and the fourth had a carcinoid in the middle section of the ileum. It is obvious that the bleeding in these cases had ceased before the tube tip had passed through the diseased area and that the presence of blood in the faeces for several days had proved misleading.

In the group where no blood was detected in the aspirates there remain six patients in whom the origin of the bleeding could not be ascertained. One of these patients died one year later and the necropsy showed peritoneal carcinosis but the primary tumour was not identified. One patient had chronic pancreatitis and cirrhosis of the liver and was too ill for laparotomy. The other four patients were followed up eight to nine years after intubation. In three of them there had been bleeding on several occasions and laparotomy had been inconclusive and the fourth had not had renewed bleeding during the period of observation.

In four patients not reported in the tables intubation could not be performed, in three of them because of lack of cooperation from the patient. In the fourth case the tube tip could not be got past the pylorus, despite repeated attempts over several weeks. 


\section{Discussion}

The five patients with confirmed coagulation defect, four of them cases of von Willebrand's disease, present a dilemma. Where there is unexplained gastrointestinal bleeding it is important to enquire about any abnormal tendency for bleeding (menstruation, shaving, wounds) in the patient and his family. However, we cannot say how often the history affords a guide to a diagnosis of a coagulation defect, since it is only in the last few years that we have consistently recorded the relevant information. A blood examination at the coagulation laboratory is made before blood transfusion or at least three weeks afterwards. It is a costly procedure, and if the history affords no grounds for suspecting a coagulation defect, it is probably justified to postpone it until a thorough examination by other methods, including intubation, has proved negative. Before explorative laparotomy, however, a major coagulation examination should be carried out. In the following discussion the cases of coagulopathy are disregarded.

From the present series it is evident that longstanding or repeated bleeding at short intervals points to a malignant tumour or haemangiomatosis, though benign polypous formations cannot be ruled out. The type of bleeding thus provides no reliable guide to the nature of its source.

In only one case did the intubation procedure produce an apparently false positive result. In this patient a positive guaiac test was obtained for specimens from the distal portion of the ileum. At laparotomy one year later changes typical of regional enterocolitis were seen in the ascending and transverse colon. It is tempting to believe that the presence of blood in the distal part of the ileum was not an artefact but due to inflammatory changes in the ileum that were not found at the operation. The possibility of a false positive result on intubation with our polyvinyl tube may thus be disregarded.

False negative results were obtained in at least four patients. The bowel contents were free of blood even below the level of a source of bleeding found later. A positive guaiac test on faeces misled us into believing that the bleeding was still occurring and we inferred, incorrectly from the results of intubation, that the bleeding was located distally to the tube.

Intubation is, of course, pointless if the bleeding has ceased. It is important not to rely entirely on faecal examination, which can give a positive guaiac test more than a week after the bleeding has ceased. If all the aspirates have been free from blood when the tip has reached the distal portion of the ileum it is therefore best to adopt one of the following two courses. Either to clean the colon with one or two large enemas and take aspirate specimens several times a day until fresh faeces are obtained, or to administer by mouth carmine chromium sulphate, or some other unabsorbable substance, that is readily observable in the faeces, and to continue ${ }_{\Omega}$ sampling the aspirate until the labelling substance

appears in the stools.
If, after one of these procedures, the faeces still contain blood while the aspirates are alle negative only then is it justifiable to conclude $\frac{\bar{c}}{\mathrm{~s}}$ that the source of bleeding lies distally to the $\frac{\bar{\sigma}}{\bar{\sigma}}$

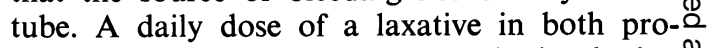
cedures can considerably shorten the intubation time. The explanation of all the false negative $\vec{\circ}$ results of intubation in our series is that we unfortunately did not observe the above rules. $\vec{\sigma}$

The intubation procedure was often mosto time-consuming. In the case of negative samples?

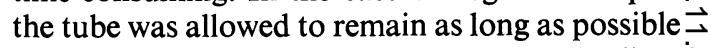
in an attempt to detect any recurrence of bleeding, and heparinization was sometimes used to ${ }_{-}$ prolong the intermittent bleeding. In the case ${ }_{0}$ of positive aspirates a lengthy confirmatory technique was used, whereby the tube was $\vec{\supset}$ repeatedly withdrawn some $20 \mathrm{~cm}$ to obtain negative specimens and then lowered again to $\overrightarrow{\overrightarrow{0}}$ obtain positive ones.

In this situation it might be an advantage, and an improvement in technique, to use a tube with two lumina, one of which would serve as the aspiration tube, while the other would

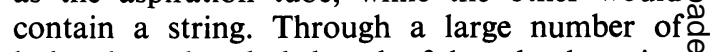
holes along the whole length of the tube the string $\stackrel{\mathbb{Q}}{2}$ would come into contact with the contents of $\overrightarrow{0}$ the bowel. A radiopaque marker, for instance 3 a clip on the string every $10 \mathrm{~cm}$, would also be useful. As soon as a positive guaiac test on an aspirate via the free lumen has been obtained $a_{C}$ general abdominal radiograph would be taken and the double-lumen tube then drawn up 3 . immediately. The site of the source of bleeding would be established by comparing the most cranial blood level on the string with the radio-o graphs. The great risk of obtaining a false positive result with an unprotected string iso avoided by enclosing the string in a soft, smooth polyvinyl tube of larger calibre than the string.

\section{Conclusions}

We recommend the following procedure for the? examination of patients with melaena in whom the history and morbid picture give no lead as to the site of bleeding.

A careful history of any exceptional tendency $\frac{\mathbb{Q}}{\mathbb{Q}}$ for bleeding should be noted, for instance, duringo shaving or menses, and also in the patient's relatives. Where there is the slightest suspiciono of haemorrhagic diathesis the coagulation laboratory should be consulted. Before diagnostiç laparotomy, coagulation defects should have been excluded where possible. Insert a tube and obtain specimens of the gastric contents. If 
blood is found, remove the tube and perform an endoscopic and radiological examination of the oesophagus, stomach, and bulb. If no blood is found in the stomach, continue the intubation until the source has been located. The next step would be to perform rectosigmoidoscopy, and then a colon radiograph, preferably by the double-contrast technique. Endoscopic and radiographic examinations of the oesophagus, stomach, and small intestine should be made, and if possible follow this immediately by angiography of the cranial mesenteric, and, possibly, the coeliac and caudal mesenteric arteries. If the bleeding has not already been sited then intubation of the small intestine to obtain bowel aspirates should follow. If this is positive then the source of bleeding can be accurately located. After this the radiographic examination of the site of bleeding can be repeated. In the case of negative specimens being obtained enemas, or orally administered dyes, are given in a further attempt to locate the site. If any of the above measures has indicated the location of the site of bleeding, laparotomy may be performed immediately. Otherwise, it should be done only during bleeding.

If the bleeding should have ceased before the source has been found, all the radiographic and endoscopic examinations should be completed and, in the case of negative results of these examinations, the patient be instructed to contact the hospital without fail should bleeding recur. If this happens within a few months, then intubation should be performed immediately to locate the source of bleeding, possibly with heparinization. Or perform diagnostic laparotomy-also without delay. Any further timeconsuming radiological examination in this situation is inadvisable, as the bleeding may again stop before the source has been found.

\section{References}

Abrams, A., and Jarvis, G. O. (1912). A new method of duodenal intubation; a preliminary note. Arch. Diagn., 5, 329-334.

Bannerman, R. M. (1957). Measurement of gastro-intestinal bleeding using radioactive chromium. Brit. med. J., 2, 1032-1034.

Baum, S., Nusbaum, M., Blakemore, W. S., and Finkelstein, A. K. (1965). The preoperative radiographic demonstration of intra-abdominal bleeding from undetermined sites by percutaneous selective celiac and superior mesenteric arteriography. Surgery, 58, 797-805.

Birke, G., and Engstedt, L. (1956). Melena and hematemesis. A follow-up investigation, with special reference to bleeding of unknown origin. Gastroenterologia (Basel), $85,97-115$.

Bockus, H. L., Ferguson, L. K., Thompson, C., and Roth, J. L. A. (1953). Management of gastrointestinal hemorrhage of undetermined origin. Value of immediate exploratory laparotomy during active bleeding. J. Amer. med. Ass., 152, 1228-1230.
Boijsen, E., and Reuter, S. R. (1967). Angiography in diagnosis of chronic unexplained melena. Radiology, 89, 413-419.

Crosby, W. H., Jr., Crosby, W. H., Sr., and Kugler, H. W. (1960). An instrument for serial sampling of intestinal juice. The intestinal cup: a means of locating the site of intestinal bleeding. Amer.J. dig. Dis., 5, 213-216.

Ebaugh, F. G., Jr., Clemens, T., Jr., Rodnan, G., and Peterson, R. E. (1958). Quantitative measurement of gastrointestinal blood loss. 1. The use of radioactive $\mathrm{Cr}^{51}$ in patients with gastrointestinal hemorrhage. Amer. J. Med., 25, 169-181.

Einhorn, M. (1909). A new method of recognizing ulcers of the upper digestive tract and of localizing them. Med. Rec. (N.Y.), 75, 549-552.

Ferguson, L. K. (1959). The diagnosis and management of bleeding from the 'lower' intestine. Surgery, 45, 352-354.

Gerritsen, T., Heinz, H. J., and Stafford, G. H. (1954). Estimation of blood loss in hookworm infestation with $\mathrm{Fe}^{60}$ Science, 119, 412-413

Green, H. L. (1964). A new suction tube for diagnosis of obscure gastrointestinal bleeding. Gastroenterology, 46, 128-133.

Halpern, M., Turner, A. F., and Citron, B. P. (1968). Hereditary hemorrhagic telangiectasia. An angiographic study of abdominal visceral angiodysplasias associated with gastrointestinal hemorrhage. Radiology, 90, 1143-1149.

Harden, W. G. S. (1960). Examination of the small bowel. In Modern Trends in Diagnostic Radiology (Third Series), edited by J. W. McLaren, pp. 84-87. Butterworth, London.

Haynes, W. F., Jr., Pittman, F. E., and Christakis, G. (1960). Location of site of upper gastrointestinal tract hemorrhage by the fluorescein string test. Surgery, 48, 821-827.

Jones, F. A., Read, A. E., and Stubbe, J. L. (1959). Alimentary bleeding of obscure origin. A follow-up study and commentary. Brit. med.J., 1, 1138-1142.

Liotta, D. (1955). Pour le diagnostic des tumeurs du pancréas: la duodénographic hypotonique. Lyon chir., 50, 455-460.

Mackay, R. S. (1968). Radioactivity and bleeding site. In BioMedical Telemetry, pp. 180-184. Wiley, New York.

Miller, T. G., and Abbott, W. O. (1934). Intestinal intubation: a practical technique. Amer. J. med. Sci., 187, 595-599.

Pesquera, G. S. (1929). A method for the direct visualization of lesions in the small intestines. Amer. J. Roentgenol., 22, 254-257.

Pillow, R. P., Hill, L. D., Ragen, P. A., Siemsen, J. S., and Walker, L. A. (1962). Newer methods for localization of obscure small-bowel bleeding. J. Amer. med. Ass., 179, 23-26.

Pittman, F. E. (1964). The fluorescein string test. An analysis of its use and relationship to barium studies of the upper gastrointestinal tract in 122 cases of gastrointestinal tract hemorrhage. Ann. intern. Med., 60, 418-429.

Pygott, F., Street, D. F., Shellshear, M. F., and Rhodes, C. F. (1960). Radiological investigation of the small intestine by small bowel enema technique. Gut, 1, 366-370.

Reuter, S. R., and Bookstein, J. J. (1968). Angiographic localization of gastrointestinal bleeding. Gastroenterology, 54, 876-883.

Rives, J. D., and Emmett, R. O. (1954). Melena: a survey of two hundred and six cases. Amer. Surg., 20, 458-470.

Roche, M. Perez-Gimenez, M. E., Layrisse, M., and Di Prisco, E. (1957). Study of urinary and fecal excretion of radioactive chromium $\mathrm{Cr}^{51}$ in man. Its use in the measurement of intestinal blood loss associated with hookworm infection. J. clin. Invest., 36, 1183-1192.

Smith, V. M. (1961). String impregnation test ('string test') for lesions of the upper digestive tract. Ann. intern. Med., 54, 16-29.

Stage, P., and Banke, L. (1968). Hypotonic duodenography. (Danish), Nord. Med. 79, 710-713.

Stahlgren, L. H., and Ferguson, L. K. (1958). The surgical management of massive melena. Amer. J. Surg., 96, 515-521.

Traphagen, D. W., and Karlan, M. (1958). Fluorescein string tes for localization of upper gastrointestinal hemorrhage. Surgery, 44, 644-645.

Valtonen, E. J. (1967). A new modification of the fluorescein string test for rapid localization of upper gastrointestinal bleedings. Gastroenterologia (Basel), 107, 216-222.

Welin, S. (1958). Modern trends in diagnostic roentgenology of colon. The Mackenzie Davidson Memorial Lecture. Brit. J. Radiol., 31, 453-464. 